\title{
Nils Bernstein und Charlotte Lerchner (Hrsg.): Ästhetisches Lernen im DaF-/DaZ-Unterricht. Literatur - Theater - Bildende Kunst - Musik -
} Film.

Universitätsverlag Göttingen 2014, 382 Seiten. ISBN:

978-3-86395-183-2

Sabine Dengscherz

Die in Buchrezensionen vertretenen Ansichten und Meinungen sind die der jeweiligen Rezensentinnen und Rezensenten und reflektieren nicht notwendigerweise die Position von SCENARIO.

Das Potenzial ästhetischen Lernens könnte im Fremd- und Zweitsprachenunterricht noch viel stärker genützt werden. Dies ist der Grundtenor des von Nils Bernstein und Charlotte Lerchner herausgegebenen Sammelbandes „Ästhetisches Lernen im DaF-/DaZ-Unterricht", in dem auch gleich viele Möglichkeiten aufgezeigt werden, wie dieses Potenzial ausgeschöpft werden könnte. Der Band geht auf einen DaF-Kongress im März 2013 an der Universidad Nacional Autónoma de México (UNAM) in Mexiko-Stadt zurück. Die Buchpublikation wurde um die Dimension DaZ erweitert und versammelt 25 Beiträge mit theoretischen Überlegungen, empirischen Forschungsergebnissen und vor allem vielen praktischen Anregungen zum ästhetischen Lernen im DaF/DaZ-Unterricht.

Dabei wird Ästhetik nicht nur im engen Sinne einer ,allgemeinen Theorie des Schönen", sondern auch in der ursprünglich viel weiter gefassten Bedeutung des altgriechischen Begriffs aisthesis verstanden, der „erst einmal nur wertfrei Wahrnehmung" meint (vgl. u.a. den Beitrag von Susanne Even, S. 192). So können sinnliche Wahrnehmung und die damit einhergehenden Emotionen, Gedanken, Eindrücke als Voraussetzung für die Auseinandersetzung mit künstlerischen Qualitäten analysiert und fruchtbar gemacht werden. Der Band ist in Abschnitte zu Literatur, Theater, Bildende Kunst, Musik und Film gegliedert, wobei die Grenzen zwischen diesen Bereichen nicht immer klar zu ziehen sind, ebenso wenig wie die Grenzen zwischen „Hoher Kunst“ und „Ästhetik im Alltag“. 
Der umfangreichste Abschnitt im Sammelband ist jener zur Literatur. Hier plädiert Carmen Schier für ästhetische Bildung als einen Wert an sich und für einen „Raum für emotional geprägte sinnliche Erfahrung und den Austausch darüber" sowie "Gelegenheiten für den eigenen gestaltenden Ausdruck" (S. 8), z.B. mittels der Erstellung von Masken, Hörbüchern oder Fotostrecken. Dazu passt auch die Position von Michael Dobstadt und Renate Riedner, die in ihrem Beitrag die Rolle von symbolic Competence und literarischer Kompetenz im Diskurs um den Kompetenzbegriff kritisch diskutieren und vor einer übermäßigen Instrumentalisierung ästhetischen Lernens warnen.

Es folgen einige konkrete Unterrichtsvorschläge: So stellt Julia Collazo ein Podcast-Projekt vor, bei dem eine Gruppe von Studierenden in Mexiko aus Rafik Schamis Erzählung „Eine deutsche Leidenschaft namens Nudelsalat“ ein gemeinsames Hörspiel erstellt hat. Heidi Hahn zeigt in ihrem Beitrag exemplarisch, wie mit Peter Schössows Bilderbuch-Adaption von Heinrich Heines Erzählgedicht „Der arme Peter“ didaktisch gearbeitet werden kann, in dem mehrere Aspekte einbezogen werden, die das Bilderbuch interessant machen: so werden Gegenwartsbezug und literarhistorischer Kontext sowie intermediale Aspekte (Text und Bild, Comic-Kunst und Theater) analysiert. Almut Hille und Johann Georg Lughofer diskutieren Poetry Slam im DaFUnterricht, eine Kunstform, die noch viel Potenzial birgt, nicht zuletzt im Hinblick auf die Motivation, Texte immer wieder zu überarbeiten. Christiane Hochstadt wiederum zeigt exemplarisch anhand der Arbeit mit Rilkes PantherGedicht, wie mimetisch-ästhetisches Lernen die Lust an der Sprache weckt und fördert, indem der Klang von Wörtern für Lernende zu einer ästhetischen Erfahrung wird, die sie genießen und die dazu führt, dass die Wörter auch in ihrer morphologischen Form - besser erinnert werden. Diesen Aspekt nützt auch Angelika Weber, die einen Grammatikunterricht skizziert, der beim induktiven Entdecken von Grammatikregeln ästhetische Aspekte einbezieht und so die Phantasie und Kreativität der Lernenden anregt: Gedichte werden nach dem Scaffolding-Prinzip zum Gerüst für eigene Textproduktion, durch Wiederholung und Variation werden Sprachstrukturen gefestigt.

Für Literatur braucht es u.a. Lesekompetenz. Antje Dohrn stellt ein Modell für literaturgestützte DaZ-orientierte Leseförderung vor, das sich nicht auf Lesemotivation beschränkt, sondern Sprachdidaktik und Literaturdidaktik verknüpft und Lesen als komplexen kognitiven Prozess begreift, bei dem Schüler*innen auf vielfältige Weise unterstützt werden können. Anhand des Beispiels von Fabeln rund um Fuchs und Rabe stellt sie konkrete Aufgaben vor für erste Textbegegnung, sukzessive Texterschließung, Textverstehen auf mehreren Ebenen sowie die eigene Textproduktion.

Einige Beiträge verorten (literarische) Texte in ihrer Beziehung zu anderen Künsten und geben Anregungen, wie mit dieser intermedialen Vernetzung im Unterricht gearbeitet werden kann. So geht Dietrich Rall der Bedeutung der Farbe Blau in der deutschen Sprache und in Literatur und Kunst nach und gibt Anregungen dafür, wie literarische Texte die Arbeit mit einem Lehrbuch ergänzen und bereichern können. Ulrike Pleß zeigt, wie mit der 
Übersetzung von Liedern im DaF-Unterricht gearbeitet werden kann und wie der Übersetzungsprozess zu einem vertiefenden Textverstehen führt. Dazu kommt die ganzheitliche Wahrnehmung von Musik und Text als eine ästhetische Einheit. Anke Stöver-Blahak wiederum stellt ein Kurskonzept vor, bei dem Schritt für Schritt das selbstständige Interpretieren und Vortragen von Gedichten erarbeitet wird und nähert sich damit bereits der Dramapädagogik an, die den zweiten Schwerpunkt des Bandes bildet.

Auch der Abschnitt zum Theater ist relativ umfangreich. Siegfried Böhm verortet dramapädagogische Ansätze als konstruktivistisch sowie handlungsund erfahrungsbasiert und zeigt damit Möglichkeiten selbst gesteuerten Lernens auf, bei dem nicht nur die vier Fertigkeiten, sondern auch nonverbales Verhalten trainiert werden. Manfred Schewe verortet das Theater und damit verbundenes performatives Lernen als Grundpfeiler für „ein didaktisches Gebäude“, in dem „das Ästhetische einen angemessenen Ort hat“ (S. 175). Das Theater kann als „vermittelnde Kunstform [... E Elemente aus anderen Künsten leicht integrieren" (ebd.), es eigne sich als Schnittstelle und könne, wie schon im Bauhaus eine Brückenfunktion einnehmen.

Allerdings sind „performativ-ästhetische Arbeitsformen im Bereich der universitären Ausbildung sowie der Fort- und Weiterbildung fremdsprachlicher Lehrpersonen nach wie vor nur in unzureichendem Maß integriert " (S. 187), wie Micha Fleiner in einer empirischen Studie herausgefunden hat. Dies sei umso erstaunlicher, als der Unterrichtssituation an sich bereits gewisse theatrale Elemente innenwohnen und der mehrdimensionale Charakter der Dramapädagogik „ein beachtliches Potenzial für die ästhetische Gestaltung fremd- und zweitsprachlicher Kommunikationsprozesse" (S. 186) darstellt. Allerdings ist nicht jede Unterrichtsaktivität, die performative Elemente enthält, auch schon Dramapädagogik oder Theaterpädagogik, denn oft werden die sinnlichen und ästhetischen Potenziale gar nicht ausgeschöpft. Dementsprechend plädiert Birgit Oelschläger dafür, auch im DaF-Unterricht Theater „deutlich von Rollenspielen und anderen Formen der Simulation zu unterscheiden" (S. 208) und nicht halbherzig zu präsentieren, sondern auch auf die Publikumswirksamkeit und die „Intensivierung der Darstellung durch bewusste Gestaltung“ (S. 213) zu fokussieren. Dabei geht es nicht um Perfektionismus; Fehler sind natürlicher Teil des Prozesses, und die Angst vor Fehlern soll überwunden werden, sowohl im Hinblick auf die Fremd- bzw. Zweitsprache, als auch im Hinblick auf die Bühne. Dabei können u.a. spielerische Übungen hilfreich sein, wie sie von Magdalena Hafner und Annemarie Kuhfuß vorgestellt werden, die außerdem das Potenzial des Improvisationstheaters für einen kommunikativen Fremdsprachenunterricht analysieren: Den Lernenden soll „ein Raum gegeben werden, in dem ohne schulischen Beurteilungszwang experimentiert werden kann und in dem ihnen ein spielerischer Zugang zu neuen Wahrnehmungsmöglichkeiten eröffnet wird“ (S. 220).

Performative Techniken können aber auch die Auseinandersetzung mit literarischen Texten vertiefen oder gezielt für die Arbeit mit Lexik verwendet werden. So reflektiert Maik Walter über Potenziale und Grenzen szenischer 
Wortschatzarbeit und präsentiert Techniken dafür, wie etwa das gesprochene Bühnenbild. Und Susanne Even stellt ein Unterrichtsmodul vor, in dem sie Lernenden auf B2-Niveau mit dramapädagogischen Methoden (Standbilder, Hotseating, Improvisation o.ä.) Conrad Ferdinand Meyers Ballade „Die Füße im Feuer" nahebringt und erlebbar macht.

Bildende Kunst, Musik und Film sind in kleineren Abschnitten mit jeweils zwei bis drei Beiträgen vertreten. Im Bereich Bildende Kunst reflektiert Eva Veronika Chen über die Bedeutung von visual Literacy für DaF-Lehrende und zeigt, wie eine Lesekompetenz für die „Sprache der Dinge“ entwickelt werden kann (vgl. S. 265) und Bilder und Objekte (Alltagsgegenstände ebenso wie Kunstwerke) nicht nur in ihrer Materialität, sondern auch auf den Ebenen ihrer aktuellen Verwendung sowie der Möglichkeitsräume, die sie eröffnen, begriffen werden können. So findet Kontextualisierung auf mehreren Ebenen statt, durch die kulturelles Sehen geübt wird. Studierenden als künftigen Lehrenden sollen damit Strategien vermittelt werden, die sie „beim eigenständigen Entdecken und Lesen ihrer Umwelt unterstützen“ (S. 269), damit sie später auch ihre Lernenden bei der Entwicklung von visual Literacy begleiten können. Ein Beispiel für die Entwicklung von visual Literacy anhand von Bildern des deutschen Künstlers Gerhard Richter bringt Tristan Lay in seinem Beitrag. Er geht dabei u.a. auf Unschärfe als ein Stilprinzip ein, das Freiräume der Interpretation eröffnet und zeigt in den Übungen auch Möglichkeiten auf, wie die Lernenden die Bilder an ihre eigene Lebenswirklichkeit anknüpfen können.

Vorschläge für die Arbeit mit Musik machen Camilla Badstübner-Kizik und Matthias Perner in ihren Beiträgen, während Katrin Wild sich mit empirischen Belegen zum Einfluss von Musik auf das Fremdsprachenlernen beschäftigt und in einer eigenen empirischen Studie mit britischen Germanistik-Studierenden im ersten Studienjahr (Niveau A2-B1) u.a. nachweist, dass sich das Spielen eines Instruments - und noch mehr das Singen - positiv auf die Aussprache auswirkt: „musikalische“ Studierende machen weniger Wortakzentfehler. Auch gezielte Rhythmusübungen konnten die Aussprache leicht verbessern (der Unterschied zur Kontrollgruppe ist aber nicht signifikant).

Camilla Badstübner-Kizik zeigt anhand der verschiedenen medialen Erscheinungsformen der Dreigroschenoper (Text, Musik, szenische Aufführung, Film), wie durch eine Verknüpfung von medialem und ästhetischem Lernen „die Reflexion der eigenen Wahrnehmung" (S. 299) in Bezug auf verschiedene mediale Erscheinungsformen von Kunstwerken angeregt werden kann: „Was kann ein literarischer Text, ein Musikstück, ein Bild besonders gut und was kann er in Bezug auf die durch ihn übermittelte Botschaft eben nicht? Durch welche Mittel prägt er/es die von ihm übermittelte Botschaft?" (S. 309)

Matthias Perner wiederum konstatiert enge Bezüge zwischen Sprache und Musik und stellt - darauf aufbauend - ein unkonventionelles Seminarkonzept vor, in dem das Genre RaP dazu dient, Sprechhemmungen abzubauen, Sprachmelodie und Sprachrhythmus zu üben.

Das Thema Film schließlich ist durch zwei Beiträge vertreten: Tina Welke zeigt anhand des Kurz(spiel)films „Hood“ (Sylvia Dahmen, 2001), einer Adaption 
des Rotkäppchenstoffs zu einem „modernen Schauermärchen“, exemplarisch, wie mit filmischen Kurzformen (z.B. Titelsequenzen, Filmausschnitten, Trailern), im DaF-Unterricht gearbeitet werden kann und Informationsfülle wie ästhetische Aspekte des Films (u.a. durch „Strategien der Verlangsamung im Rezeptionsprozess“, S. 361) in ihrer Wechselwirkung analysiert werden können. Und Mitherausgeber Nils Bernstein schließlich geht in seinem Beitrag auf zwei Filme über den RAF-Terror („Wer wenn nicht wir“ und „Der Baader-MeinhofKomplex") ein und zeigt, wie sich im DaF-Unterricht anhand von verfilmten historischen Ereignissen die „Fiktion des Faktischen“ (S. 373) thematisieren und analysieren lässt, mit dem Ziel, eine kritische Medienkompetenz zu fördern, die Lernende dazu befähigt, „bei der Rezeption des mannigfaltigen Angebots an Informationsquellen“ bewusst, ,ein eigenes, zwangsweise berechtigt subjektives Bild von vergangenen Sachverhalten zu erhalten" (S. 374).

Insgesamt bietet der Band eine breite Palette fundierter theoretischer Auseinandersetzung mit der Rolle sinnlich-ästhetischer Wahrnehmung im DaF/DaZ-Lernen und eine Fülle an Vorschlägen für die praktische Umsetzung. Es ist wahrscheinlich überzogen, nach der kommunikativen und der kognitiven gleich eine ästhetische Wende einzuläuten, aber ein Grundstein ist sicherlich gelegt dafür, dass ästhetisches Lernen und sinnliche Wahrnehmung breiterer Raum im Unterricht und in der Lehrer*innen-Aus- und Fortbildung eingeräumt wird. Es gibt hier sicherlich noch viel zu entdecken. 